\title{
Producción de dos cepas de Frankia sp. aisladas de Alnus acuminata H.B.K. por fermentación fed-batch
}

\section{Production of two strains of Frankia sp. isolated from Alnus acuminata H. B. K. using fed-batch fermentation}

\author{
Produção de duas estirpes de Frankia sp. isolado \\ a partir de Alnus condiloma H.B.K. por fermentação \\ em batelada alimentada
}

Ana María Rey Obando ${ }^{1}$, Rolando Barahona Rosales ${ }^{2}$ \& Diego Rosendo Chamorro Viveros Abierta y a Distancia UNAD. Medellín.

1ana.reyob@ucc.edu.co, ${ }^{2}$ rbarahonar@unal.edu.co, ${ }^{3}$ diego.chamorro@unad.edu.co

\section{Resumen}

El nitrógeno, uno de los elementos de mayor importancia en los sistemas de producción agropecuario, es aportado al suelo a través de fertilización química, fenómenos naturales y fijación biológica por microorganismos del suelo como Frankia, un mecanismo ambiental y económicamente viable. El uso de Frankia como inoculante exige un profundo estudio relacionado con el desarrollo y análisis del comportamiento de los inóculos en condiciones controladas. Se realizaron evaluaciones enfocadas en estudiar y estandarizar las condiciones que permitieran el desarrollo de un prototipo de inoculante de Frankia a base de las cepas Aan17, Aac49 y la cepa Chilena Aaclll, antes de su producción a gran escala. Se determinaron las condiciones nutricionales que influyen sobre el crecimiento y formación de estructuras especializadas de Frankia en un sistema de fermentación fed-batch a escala de un litro.
La dinámica del crecimiento en los medios evaluados mostró diferencias altamente significativas $(P<0.001)$ observándose la mayor velocidad especifica de crecimiento $\left(0.025 \mathrm{~h}^{-1}\right)$ y menor tiempo de duplicación ( 33.9 h) en el medio M1 extracto de raíz, levadura y glucosa. Con el medio M3 extracto de raíz, levadura y melaza, se observó el mayor tiempo de duración de la fase exponencial $(64.86$ h), la mayor velocidad específica de consumo de sustrato (0.007) y el mayor rendimiento celular con base al sustrato ( $1.95 \mathrm{~g} /$ proteína/sustrato). Se evidenció que los componentes nutricionales evaluados presentaron influencia sobre la producción de biomasa $(P<0.05)$, mostrando mayor producción de biomasa respecto al medio de referencia Benzil Amina Purina (BAP) $(P<0,01)$.

Palabras clave: microsimbiontes, diazotrofos, inoculantes, fermentación 


\section{Abstract}

Nitrogen, one of the most important factors in the agricultural production systems, it is provides to the ground through chemical fertilization, natural phenomena and biological fixation by soil microorganisms as Frankia, a mechanism environmentally and economically viable. The use of Frankia as inoculant requires a thorough study related to the development and analysis of the behavior of the inocula in controlled conditions. Assessments were carried out focused on study and standardize the conditions that allow the development of a prototype of inoculant to base of Frankia strains Aan17, Aac49 and eca Aaclll Chilean, prior to its production to large scale. It was determined the nutritional conditions that influence the growth and formation of specialized structures of Frankia in a fermentation fed-batch system on the scale of a liter. The dynamics of growth in the media evaluated showed highly significant differences $(P<0.001$ ) observing the highest specific growth rate $(0,025$ $\mathrm{h}-1)$ and shorter doubling time (33.9 h) in the medium M1 root extract, yeast and glucose. With the average M3 root extract, yeast and molasses, it was noted the widest length of time of the exponential phase $(64.86 \mathrm{~h})$, the highest specific speed of substrate consumption $(0,007)$ and the largest cellular performance based on the substrate (1.95 $\mathrm{g} /$ protein/substrate). It was evidenced that the nutritional components evaluated presented influence on the production of biomass $(P<0.05)$, showing higher biomass production in regard to the reference medium Denzil Amina Purina (BAP) $(P<0.01)$.

Key-words: microorganisms, diazotrofos, inoculants, fermentation

\section{Resumo}

Nitrogênio, um dos elementos mais importantes nos sistemas de produção agrícola, é fornecido ao solo através de fertilizantes químicos, fenômenos naturais e fixação biológica por microrganismos do solo como Frankia, um mecanismo ambiental e economicamente viável. Utilização de Frankia como inoculo requer um estudo exaustivo relacionada com o desenvolvimento e a análise do comportamento dos inoculos sob condições controladas. Foram realizadas avaliações focalizadas em estudar e padronizar as condições que permitiram o desenvolvimento de um protótipo de inoculante baseado em cepas Frankia Aan17 e Aac49 chilenos e Aaclll Antes da produção em grande escala. Foram determinadas condições nutricionais que influenciam o crescimento e formação de estruturas especializadas de Frankia num sistema de fermentação fed-batch em escala de um litro. A dinâmica de crescimento nos meios testados apresentaram diferenças altamente significativas $(P<0,001)$ seobservando a maior taxa de crescimento específico $(0,025$ h-1) e menor tempo de duplicação (33,9 h) no meio M1 extrato de raiz, levedura e glicose. Com o meio M3 extrato de raiz, de levedura e melaço observou-se a maior duração da fase exponencial $(64,86 \mathrm{~h})$, a maior taxa de consumo de substrato específico $(0,007)$ e o rendimento mais elevado de células com base no substrato (1,95 g / proteína / substrato). Evidenciou-se que os componentes nutricionais avaliados apresentaram influência sobre a produção de biomassa $(P<0,05)$, com maior produção de biomassa em comparação com a média de referência BenziloAmine Purina (BAP) $(P<0,01)$.

Palavras-chave: microssimbiontes, diazotroficos, inoculantes, fermentação

\section{Introducción}

Tradicionalmente, el uso del recurso suelo en los sistemas de producción es irracional, llegando a ser extractivo e intensivo, siendo la población y el crecimiento demográfico uno de los principales determinantes de la demanda de alimentos y otros productos agrícolas, con un incremento anual de 76 millones de dólares según la ONU (Steinfeld et al., 2009). Adicionalmente, varios de los procesos productivos tradicionales contribuyen con la expansión de la frontera agrícola hacia áreas 
con diversas limitaciones, desconociendo las características estructurales y químicas de los suelos (Wani, Rupela \& Lee, 1995). Esto genera alta susceptibilidad a la erosión y reduce la capacidad del suelo para sostener cultivos en condiciones óptimas de producción. Además, el incremento en el uso de los fertilizantes nitrogenados ha estado acompañado por un aumento exponencial en el consumo de formas no renovables de energía, las cuales se han convertido en un factor limitante para lograr aumentos de los rendimientos (Broom, Galindo \& Murgeitio, 2013).

Finalmente, los efectos claros del cambio climático exigen la reconversión agropecuaria hacia modelos sostenibles (Chamorro \& Rey, 2005). Es por ello que la identificación de opciones ambiental, económica y socialmente viables cobra gran importancia, dado su papel potencial en la reconversión ambiental de los sistemas de producción agropecuarios (Steinfeld et al., 2009).

El uso de la biotecnología contribuye a esta reconversión, siendo el uso de microorganismos con una gran variedad de propiedades bioquímicas y fisiológicas de interés para el hombre (Nain et al., 2010). En este sentido y con el fin de potenciar el uso de microrganismos por los procesos bioquímicos a fin de lograr el máximo aprovechamiento del material biológico bajo condiciones nutricionales y físico-químicas controladas (Gyaneshwar, Naresh, Pareh \& Poole, 2002) con el fin de optimizar la producción en bioreactores, fermentadores de pequeña escala o fermentadores a nivel industrial y al mismo tiempo, generar productos microbiales altamente competitivos en el mercado.

El uso de inoculantes comerciales a partir de cepas de microorganismos del suelo, ha reportado buenos resultados en la agricultura, lográndose suplir las necesidades de elementos esenciales para garantizar el suministro adecuado de nutrientes a las plantas (Boraste et al., 2009). Desafortunadamente, la variedad de microorganismos benéficos disponibles está limitada a un determinado grupo de especies, siendo esta una de las mayores limitantes al uso de estas tecnologías por parte de los productores. Por lo anterior, es importante realizar investigaciones que permitan mejorar las condiciones de producción de inoculantes de microorganismos altamente especializados a bajos costos de producción, permitiendo la generación de un producto innovador y competitivo en el mercado.

El actinomiceto Frankia establece asociaciones simbióticas con diversos grupos de angiospermas no leguminosas o plantas actinorrizas e induce la formación de nódulos fijadores de nitrógeno (Benson, Vanden Heuvel \& Potter, 2004; Chaia, Wall \& Huss-Danell, 2011). Las plantas actinorrízicas, constituidas por ocho familias, cuatro géneros y 200 especies, son arbustos o árboles que habitan diversos ecosistemas (Huguet, Mergeay, Cervantes \& Fernández, 2004). La asociación entre la planta y la bacteria tiene gran importancia ecológica, ya que las bacterias se adaptan a condiciones ambientales extremas y poseen una gran capacidad de crecer en suelos de baja fertilidad o después de algún disturbio, comportándose como pioneras en el desarrollo sucesional de comunidades vegetales en suelos con bajos contenidos de nitrógeno, siendo algunas de ellas de gran valor en la recuperación y protección de suelos degradados y erosionados (Dawson, 1990; Anderson, 2011; Beauchemin et al., 2011). Entre los grupos más representativos de estas especies en América del Sur se encuentran Coriarieceas, Myricaceaes y Betuláceas, a esta última familia pertenece la especie Alnus acuminata H.B.K. ssp acuminata (Romero, 1996; Chamorro \& Rey, 2008).

La presente investigación estuvo enfocada a mejorar las condiciones de crecimiento, a escala piloto de cepas nativas promisorias de Frankia aisladas de dos zonas geográficas de Colombia, que permitan determinar las condiciones de producción de un inoculante basado en Frankia. 


\section{Materiales y Métodos}

Cepas de estudio: Las cepas de Frankia Aan17 y Aac49 fueron aisladas de una población natural de Alnus acuminata H.B.K de la vereda Cubijan Alto, municipio de Pasto, departamento de Nariño, a una altura de 3.070 m.s.n.m. y una temperatura promedio de $13.5{ }^{\circ} \mathrm{C}$, y de la vereda Villa Nataly, localidad de Usme, departamento de Cundinamarca, a 3.045 m.s.n.m y $12{ }^{\circ} \mathrm{C}$. Las cepas Aan17 y Aac49 se clasificaron a nivel de género a través de pruebas bioquímicas, morfológicas y moleculares y se evaluaron en plantas del hospedero, mostrando una respuesta positiva, sinergismo que fue reflejado en un rápido crecimiento y mayor respuesta en las variables dasométricas y de composición química de $A$. acuminata durante la fase de vivero (Rey, 2006; Pintor, Garzón, Chamorro \& Rey, 2007; Chamorro \& Rey, 2008), resultados que permitieron seleccionar estas cepas como promisorias para la producción de inoculantes biológicos del suelo.

Como cepa control se empleó la cepa Aaclll aislada de nódulos de $A$. acuminata, procedentes de Argentina. La cepa fue evaluada y caracterizada por Carú, Becerra, Sepúlveda \& Cabello, (2000) en la Universidad de Chile (Área de Microbiología, Departamento Forestal).

Las cepas, conservadas en medio Benzil Amina Purina (BAP) más glicerol (500 ul BAP + 150 ul glicerol $10 \%$ ) a una temperatura de $-4^{\circ} \mathrm{C}$, fueron activadas y crecidas por un periodo de dos meses, en medio líquido BAP modificado por Murry, Fontaine \& Torrey, (1984), a una temperatura de $36{ }^{\circ} \mathrm{C}$ y agitación constante de 150 rpm (Rey, 2006). Se realizaron tinciones en fresco con azul de tripán y tinción de Gram con el fin de verificar la pureza.

Las curvas de crecimiento de las cepas fueron realizadas en medio de cultivo líquido, el cual fue BAP con Glucosa y $\mathrm{NH}_{4}{ }^{+}$como fuente de carbono y nitrógeno, a $36^{\circ} \mathrm{C}$, agitación constante (150 rpm) y mediciones diarias, usando como pará- metro de referencia el crecimiento en función de la proteína total por medio del método de Lowry (Lowry, Rosenbrough \& Randall, 1951) ajustado por Rey (2006).

Se realizó un subcultivo con un vial de $15 \mathrm{~g}$ de inóculo en $50 \mathrm{~mL}$ del medio de cultivo a evaluar en la fermentación. Estos subcultivos fueron mantenidos con agitación constante a 150 rpm y a una temperatura de $36{ }^{\circ} \mathrm{C}$ durante 72 horas a un $\mathrm{pH}$ de 6,5. Posteriormente, se vertieron $10 \mathrm{~mL}$ del inóculo que corresponde al $1 \%$ del volumen total a fermentar en $990 \mathrm{~mL}$ de cada uno de los medios de cultivo a evaluar. Se emplearon erlenmeyer con capacidad de dos litros, a los cuales se les adaptó un sistema de toma de muestra. Las condiciones en las que se llevó a cabo la fermentación fueron: periodo de incubación 17 días, $36 \stackrel{\circ}{\circ} \mathrm{C}, \mathrm{pH}$ 6,5 y agitación constante de 150 rpm.

Evaluación de la cinética de crecimiento: Se realizaron dos repeticiones durante el transcurso de la fermentación, recolectando $5 \mathrm{~mL}$ de muestra en condiciones estériles con intervalos diarios partiendo desde la hora 0 primer día hasta el día 15. En cada muestreo se determinó:

Crecimiento microbial por Lowry: Lowry et al. (1951) modificado por Rey (2006): El micelio fue obtenido por filtración en papel Whatman No. 41, realizando lavados con $10 \mathrm{~mL}$ de $\mathrm{MgSO}_{4}$ a $\mathrm{pH}$ 7. Las cepas fueron expuestas a choque térmico con nitrógeno líquido y agua caliente para inhibir el crecimiento y finalmente se llevaron a sonicación por 30 segundos. El crecimiento de las cepas en los microensayos se determinó por el contenido de proteínas totales, las absorbancias leídas (420 nm).

Reducción de acetileno (ARA). El etileno fue medido en un cromatógrafo de gas con detector de ionización de llama, la actividad reductora de acetileno se estableció en $\mu$ moles de etileno (Rediet et al., 2013). 
La cuantificación del número de vesículas por mL, se realizó mediante un lavado de las muestras con agua destilada y centrifugación a 2500 rpm adicionando el $10 \%$ del volumen con solución tween 20. Con el sobrenadante se realizó la lectura por conteo en cámara de Neubauer con profundidad de un $1 \mathrm{~mm}$. El criterio de conteo de vesículas maduras fue por refracción y presencia de talo hifal (Schultz, \& Benson, 1989; Rediet, Mansour \& Tisa, 2013). Se realizó el recuento cada dos días de la fermentación (1, 3, 5, 7, 9, 11, 13 y 15 días).

Parámetros de evaluación de los inoculantes

Con los datos obtenidos en las curvas de crecimiento se determinaron los parámetros cinéticos como rendimiento de biomasa a partir del sustrato $\mathrm{Y}_{\mathrm{x} / \mathrm{s}}$ (Proteína/g), velocidad específica de crecimiento $\mu$ $\left(\right.$ dia $\left.^{-1}\right)$, tiempo de duplicación td $(\mathrm{h})$ y productividad (Doran, 1998; Duarte, 1998). Terminada la fermentación, se realizó un análisis en un cromatógrafo de gases, con el fin de identificar los compuestos originados durante ésta. Para ello, se tomó una submuestra de $1 \mathrm{~mL}$ de la muestra inicial, se llevó a centrifugación a 13000 rpm durante 20 minutos, luego se filtró en una membrana de $0.45 \mu$ y se tomó una alícuota de $800 \mu \mathrm{L}$ más $200 \mu \mathrm{L}$ de la solución estándar 2-isobutanol. Finalmente, de esta solución se inyectaron $10 \mu \mathrm{L}$ en el cromatógrafo.

Análisis estadístico: Para realizar el análisis de varianza de la fase exponencial de los medios de cultivo en relación a cada una de las cepas y los estimados del crecimiento microbial por nitrógeno se aplicó un diseño factorial, compuesto por dos factores:

Factor A: se consideraron los medios de Cultivo Medio 1: Extracto de raíz + extracto de levadura + glucosa; Medio 2: Extracto de raíz + extracto de levadura + sacarosa; Medio 3: Extracto de raíz + extracto de levadura + melaza.

Factor B: Cepas de Frankia (Cepa Aac49, Cepa Aan17, AacllI).
La compararon de medias se realizó utilizando el Test de Tukey, con un nivel de confianza del 95\%. Los análisis fueron realizados empleando el software SAS, 9.0 para Windows

\section{Resultados y Discusión}

\section{Dinámica del crecimiento}

La cinética de crecimiento de las cepas de Frankia evaluadas en cultivo en batch, presentaron semejanzas a las observadas con otras bacterias y con hongos filamentosos. En las Figuras 1, 2 y 3, se muestran las curvas de crecimiento microbial durante el periodo de fermentación.

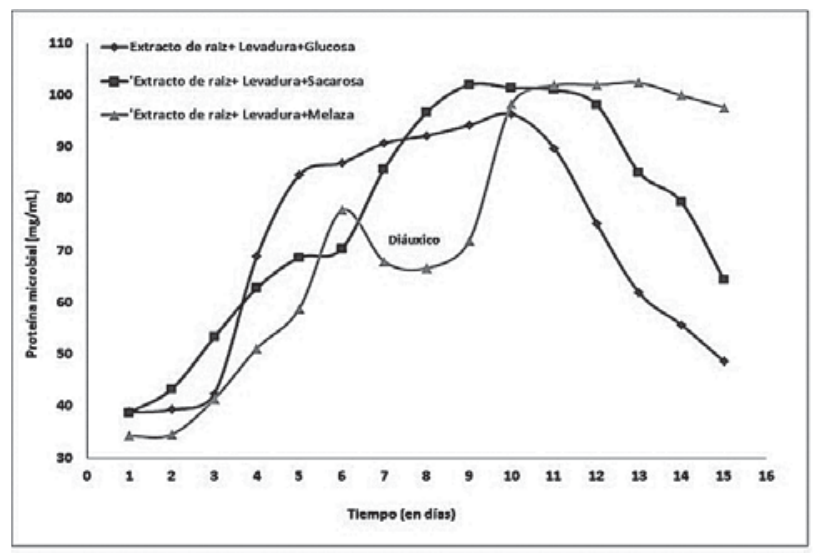

Figura 1. Curva de crecimiento microbial de la cepa Aac49 en los tres medios de cultivo evaluados

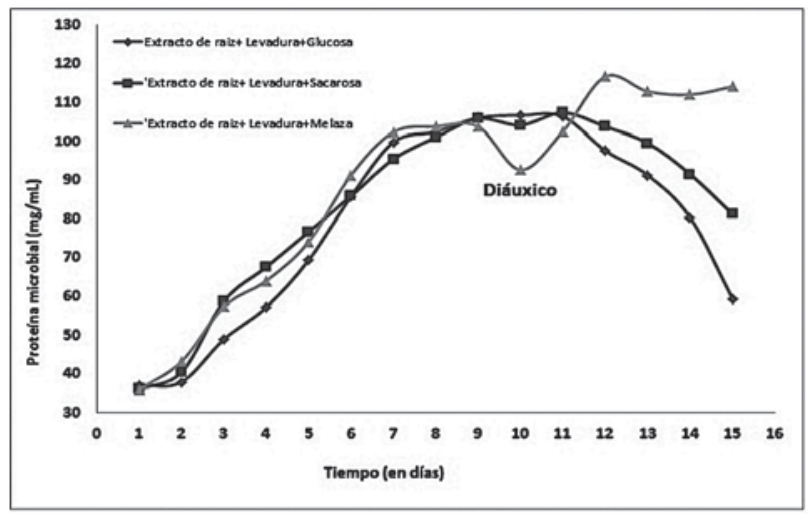

Figura 2. Curva de crecimiento microbial de la cepa Aan17 en los tres medios de cultivo evaluados 


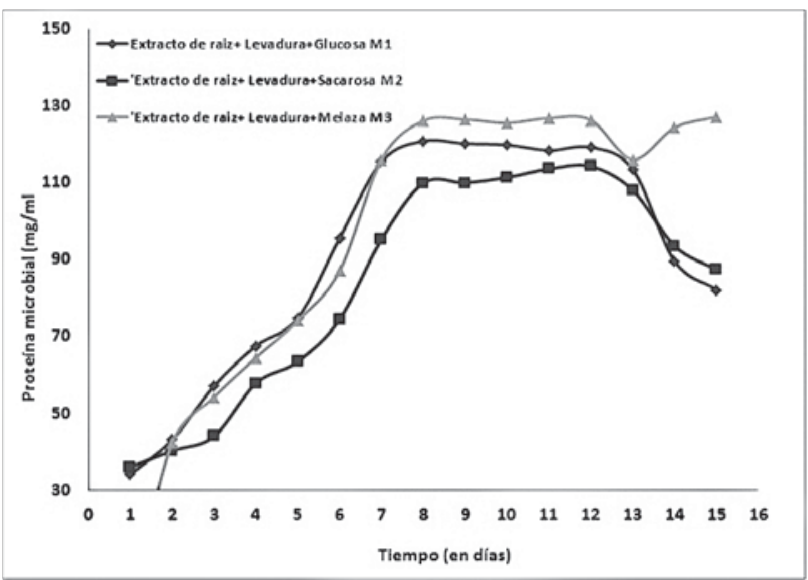

Figura 3. Curva de crecimiento microbial de la cepa Aaclll en los tres medios de cultivo evaluados

La dinámica del crecimiento de las cepas nativas de Frankia durante el proceso de fermentación, presentó una corta fase de adaptación que osciló entre uno a dos días, y que en los medios con melaza en la cepa Aan17 y sacarosa en la cepa Aac49 estuvo ausente (Figuras 1 y 2 ). Este comportamiento, pudo estar asociado a que los microorganismos cultivados provenían de un preinoculo obtenido a partir de células cultivadas bajo las mismas condiciones. La fase logarítmica se presentó en la mayoría de los casos a partir del segundo día, respuesta que no se relacionó con el tipo de medio ni con la cepa evaluada. Partiendo desde el quinto hasta el octavo día, se observó la fase estacionaria, con la mayor permanencia en esta fase observándose en los medios con melaza en las tres cepas evaluadas.

La muerte celular presentó un comportamiento diferente entre cepas, pero no entre medios, con excepción de la cepa control Aaclll que no manifestó esta fase durante el tiempo de evaluación en el cultivo M3 con melaza (Figuras 1, 2 y 3). Se observó que la muerte celular se presentó entre los días 10 y 13, periodo en el que el carbono disponible en los medios se encontraba en promedio agotado en $99 \%$ en todas las fermentaciones y la fuente de nitrógeno mostraba un promedio de agotamiento del $68 \%$.
Microscópicamente se observó autolisis celular en las cepas nativas, efecto asociado a la carencia de carbono (Murry et al., 1984). La presencia de melaza en el medio M1 (extracto de raíz, levadura y melaza) reversó el proceso de autolisis, generando una curva de crecimiento diaúxico en las tres cepas evaluadas (Figuras 1, 2 y 3), el cual ocurre como consecuencia de la represión catabólica en presencia de las fuentes de energía aportadas tanto por la melaza como el extracto de raíz.

Con respecto a la respuesta del efecto de los medios de cultivo frente a la biomasa total de cepas medido en la concentración de proteínas, se obtuvieron diferencias altamente significativas entre tratamientos, especies y tratamiento*especie $(\mathrm{P}<.0001)$ (Figura 4).

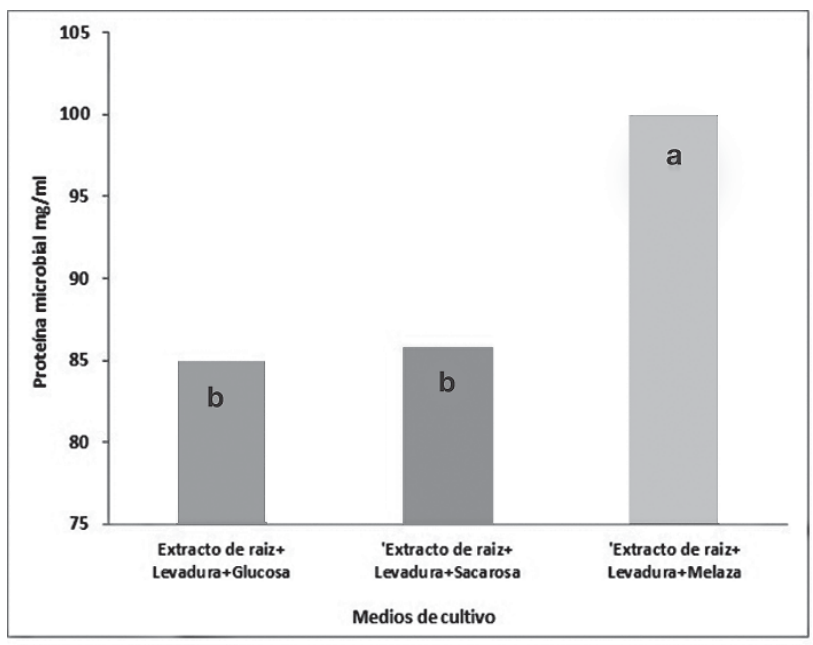

Figura 4. Efecto de los medios de cultivo sobre el crecimiento de las cepas

** Diferencias altamente significativas $(P<0.01)$. Diferencias significativas $(P<0.05)$. ns no existen diferencias Promedios en una misma columna con letras iguales no difieren significativamente según la prueba de Tukey $(P>0.05)$

El medio de cultivo con extracto de raíz, levadura y melaza, superó a todos los demás tratamientos con una producción de proteína microbiana de $100 \mathrm{mg} / \mathrm{mL}$, superando en 16,5 y $17,7 \%$ a los medios con sacarosa y glucosa. Los resultados de crecimiento microbial, estimados a través de 
la cuantificación de proteína microbial, coinciden con los reportes de Quispel \& Tak (1978), Quispel, Burggraaf, Borjse \& Tak (1983) y Wolters (1998), quienes manifiestan que la adición de extractos de plantas beneficia el crecimiento in vitro, debido a que estos poseen sustancias químicas que actúan como componentes activos y nutrientes.

En el caso de las raíces de $A$. acuminata, se presentan fenoles, minerales y compuestos orgánicos, que en el suelo inhiben de manera selectiva el crecimiento de patógenos y estimulan bioquímicamente a Frankia para su crecimiento (Wolters, 1998; Beauchemin et al., 2011).

Adicionalmente, el extracto de raíz puede ser una fuente importante de lípidos para las células, ya que se encuentra la hidroxidamarenona II (dipterocarpol) de manera activa, lo que permite estimular la producción de vesículas, conformadas en sus paredes por lípidos que participan para brindarle a la nitrogenasa las condiciones de anaerobiosis necesarias en el proceso de fijación biológica de nitrógeno Heath \& Tiffin, 2007).

De igual manera, se ha reportado que las colonias de Frankia en cultivos puros producen enzimas extracelulares, principalmente celulasas, pectinasas y proteínas que permiten emplear de manera eficiente el extracto de raíz (Benoist, Muller, Diem \& Schwenkel, 1992). Así, Quispel et al. (1983), reportaron la dependencia del extracto de raíz para el crecimiento de algunas colonias.

El aporte nutricional de las diferentes fuentes empleadas como la melaza y la levadura, permitieron encontrar caminos alternativos para la biosíntesis de estas moléculas. En este sentido, la melaza posee componentes complejos que crean la necesidad a las células de expresar una mayor variedad de enzimas catalíticas para degradar estos sustratos. Por otro lado, los aportes nutricionales de la levadura en forma de aminoácidos, vitaminas y carbohidratos solubles, mejoran el balance nutricional nitrógeno-carbono (Nahvi, Emtiazi \& Alkabi, 2002).

En cuanto al consumo del sustrato, que se muestra en las Figuras 5, 6 y 7, se observa que durante el periodo de fermentación hubo un agotamiento sucesivo de fuentes de azúcares totales y nitrógeno disponibles en los diferentes medios de cultivo durante el transcurso de la fermentación en las tres cepas evaluadas.
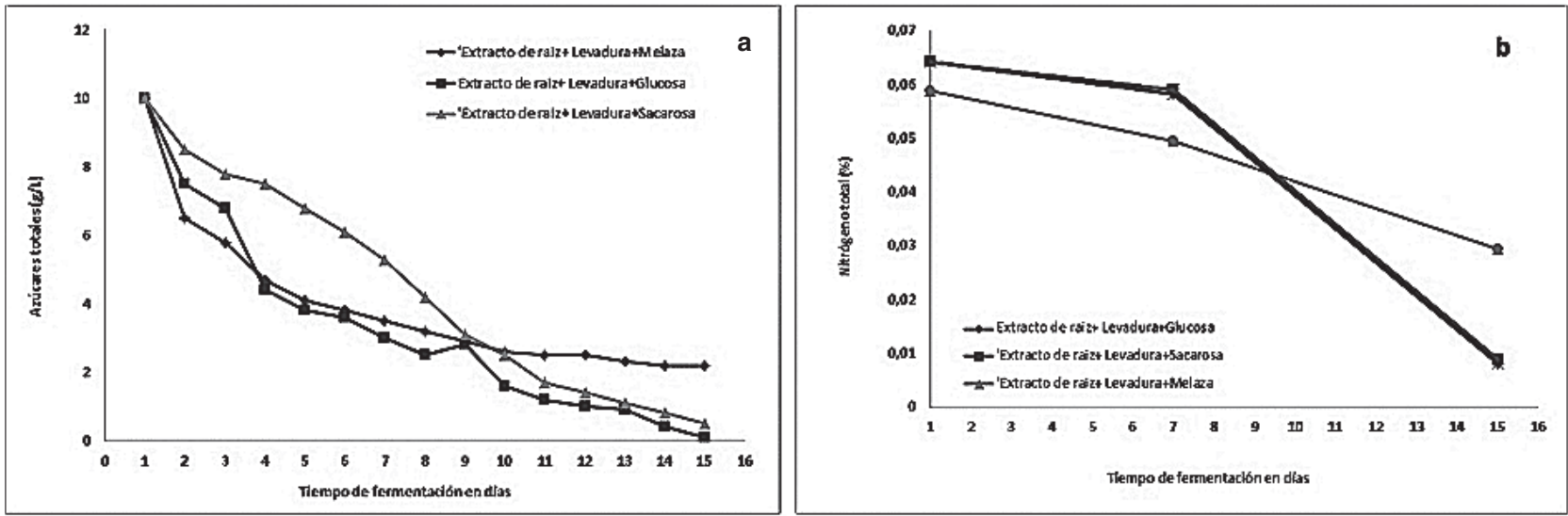

Figura 5. a). Consumo de azúcares totales (g/L) y b). Nitrógeno total (\%) de la cepa Aac49 

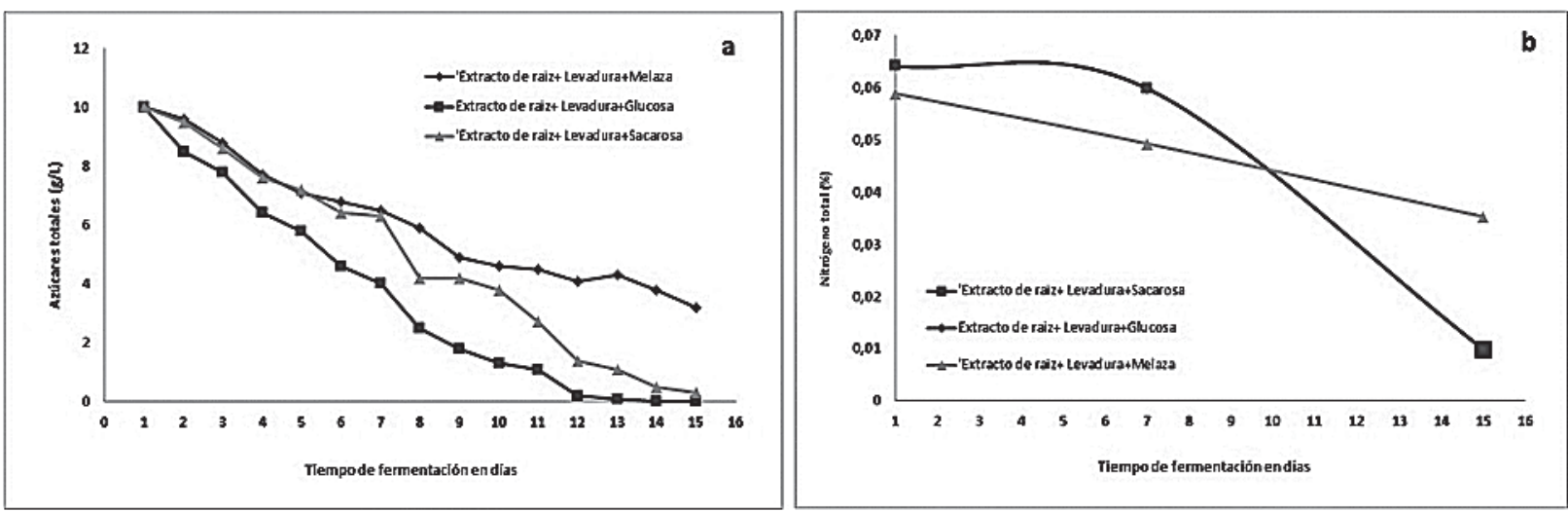

Figura 6. a) Consumo de azúcares totales ( $\mathrm{g} / \mathrm{L}$ ) y b) Nitrógeno total (\%) de la cepa Aan17
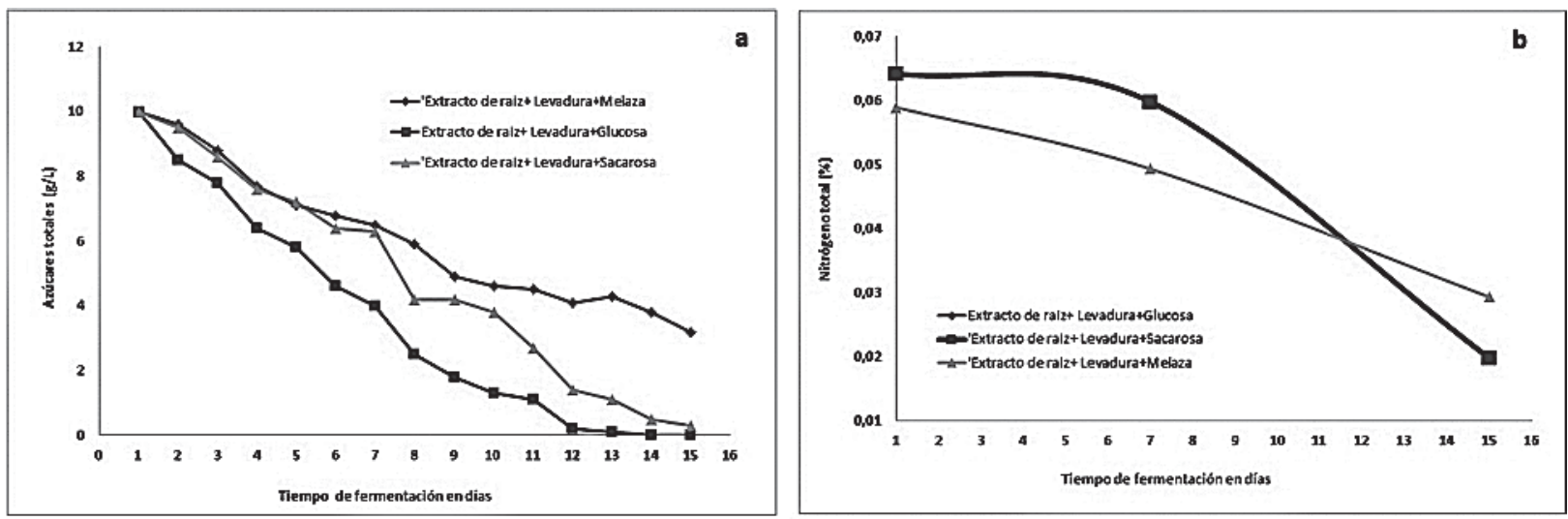

Figura 7. a) Consumo de azúcares totales (g/L) y b) Nitrógeno total (\%) de la cepa Aaclll

La factibilidad económica de algunos procesos biotecnológicos depende básicamente del costo de las materias primas utilizadas en ellos. En la práctica, la selección de un microrganismo con elevado rendimiento de sustrato en biomasa favorece la productividad del proceso. Generalmente, cuando el sustrato es el factor limitante de crecimiento, la cantidad de biomasa producida es proporcional a la cantidad de reactivo limitante consumido (Duarte, 1998). En este estudio se observó una relación típica inversamente proporcional entre la acumulación de biomasa y el azúcar y el nitrógeno residual durante la fermentación (Figuras 5, 6 y 7).

En los primeros 8 días de fermentación del medio extracto de raíz, levadura y glucosa, fueron consumidos entre un $75 \%$ a un $80 \%$ de los azúcares totales disponibles por las tres cepas, resultado que coincide con la mayor velocidad específica de crecimiento que se presentó en las cepas en este medio de cultivo. Al finalizar la fase exponencial que en promedio fue a los 13 días, quedo remanente entre el 1 y el $9 \%$ del azúcar total en todos los medios de cultivo evaluados.

El consumo de nitrógeno al final de la fase exponencial osciló entre un 7,5 a un $16,6 \%$. El mayor consumo de nitrógeno en esta fase de crecimiento fue para las cepas Aan17 y Aaclll con valores de $9,95 \%$ y $9,94 \%$, respectivamente, la cepa nativa Aac49 presentó un consumo del $7.82 \%$. Al finalizar el periodo de fermentación (día 15) se presentó un consumo del nitrógeno del $80 \%$ y $80 \%$ en las cepas creciendo en los medios de cultivo con glucosa y con sacarosa, respectivamente mientras que en el medio con melaza se presentó un consumo de nitrógeno del $46,7 \%$.

El cambio en el contenido de azúcar residual a lo largo de la fermentación se puede asociar a 
la presencia de la enzima invertasa que estimula el consumo de sustrato a medida que actúa sobre la sacarosa produciendo glucosa y fructosa, azúcares reductores que son más simples y pueden ser asimilados con mayor facilidad (Kazuhiko \& Kozo, 1995). Este proceso de inversión de la sacarosa depende de las concentraciones de glucosa en el medio de cultivo, es decir, que a mayor concentración de glucosa a una temperatura de $35^{\circ} \mathrm{C}$, se evidencia con mayor facilidad la actividad de la enzima invertasa (Schubert et al., 2011).

\section{Parámetros cinéticos}

El estudio de las fases de crecimiento permite comparar las tres cepas de Frankia en los tres medios de cultivo a escala de 1 litro (Tabla 1). La velocidad de crecimiento de un microorganismo frente a sustrato, depende de la concentración de nutrientes disponibles presentes. Es normal que a altas concentraciones de nutrientes, la velocidad específica alcance los valores máximos permitidos por la cinética intrínseca de las reacciones intracelulares, las cuales están relacionadas con la transcripción y traducción del DNA (Agudelo, Ortega \& Hoyos, 2010).

Tabla 1. Parámetros de la cinética de la fase exponencial del crecimiento de las cepas de Frankia en tres medios de cultivo a escala de 1 litro

\begin{tabular}{|c|c|c|c|c|c|c|c|c|c|c|c|c|c|}
\hline \multirow{2}{*}{ Parámetros } & \multicolumn{2}{|c|}{$\begin{array}{l}\text { Promedio } \\
\text { medios }\end{array}$} & \multicolumn{3}{|c|}{$\begin{array}{c}\text { Promedio } \\
\text { cepas }\end{array}$} & \multicolumn{3}{|c|}{ Cepa Aac49 } & \multicolumn{3}{|c|}{ Aan17 } & \multicolumn{2}{|c|}{ Aaclll } \\
\hline & $M 2^{*}$ & $M 3^{*}$ & $\begin{array}{c}\text { Aac } \\
49\end{array}$ & $\begin{array}{c}\text { Aan } \\
17\end{array}$ & Aacwlll & $\mathrm{M1}^{*}$ & M2* & $M 3^{*}$ & $M 1^{*}$ & $M 2^{*}$ & $M 3^{*}$ & $M 1^{*}$ & $M 2^{*}$ \\
\hline
\end{tabular}

$\mu$ : Velocidad

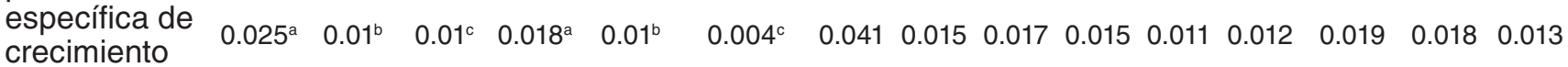
$\left(h^{-1}\right)^{\star \star}$

Td: Tiempo de duplicación (h) ${ }^{* *}$

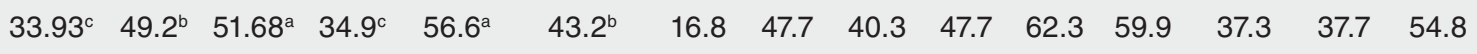

T: Tiempo de duración de la fase exponencial $(h)^{\star \star}$

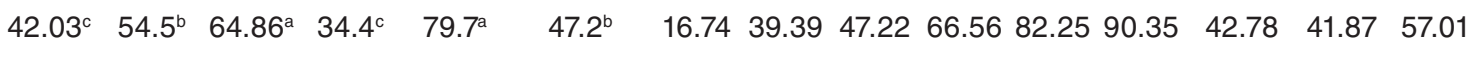

n: Numero de generaciones

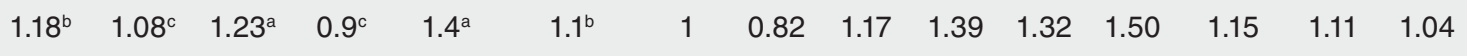

$\mathbf{q}_{\mathrm{s}}$ : Velocidad específica de consumo de

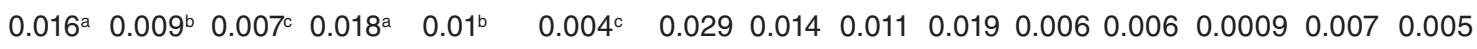
sustrato **

$\mathbf{Y}_{\mathrm{xs}}:$ Rendimiento celular en base al sustrato $\begin{array}{lllllllllllllll}1.59^{c} & 1.82^{\mathrm{b}} & 1.95^{\mathrm{a}} & 1.59^{\mathrm{c}} & 1.82^{\mathrm{b}} & 1.95^{\mathrm{a}} & 1.41 & 1.07 & 1.6 & 1.37 & 1.84 & 1.9 & 2.01 & 2.56 & 2.35\end{array}$ (g proteína/g sustrato) $^{\star *}$

* Medio 1: Extracto de raíz + extracto de levadura + glucosa; Medio 2: Extracto de raíz + extracto de levadura+ sacarosa; Medio 3: Extracto de raíz + extracto de levadura+ melaza ** Diferencias altamente significativas $(P<0.01)$. * Diferencias significativas $(P<0.05)$. ns no existen diferencias Promedios en una misma columna con letras iguales no difieren significativamente según la prueba de Tukey $(P>0.05)$ 
Al comparar la velocidad específica de crecimiento celular $(\mu)$ que se presentó en la fase exponencial, en las tres cepas de Frankia en los medios de cultivo, se observaron diferencias altamente significativas $(P<.0001)$. En la respuesta de $\mu$ de las cepas en cada uno de los tres medios de cultivos evaluados, se observó que el medio con extracto de raíz, levadura y glucosa (M1) estuvo asociado con un mayor valor de $\mu$ de $0,025 \mathrm{~h}^{-1}$. El menor $\mu$ se presentó en el medio con extracto de raíz, levadura y melaza (M3) con un valor de 0,014 $\mathrm{h}^{-1}$. En cuanto a la velocidad de crecimiento $\mu$ entre cepas de Frankia, el aislamiento Aac49 proveniente de Cundinamarca, superó a las demás cepas con un valor medio de $0,041 \mathrm{~h}^{-1}$.

El número de generaciones obtenidas a los 15 días de evaluación presentó diferencias altamente significativas entre medios y entre cepas $(P<.0001)$. Entre los medios, el medio M3 (con melaza) presentó el mayor número de generaciones con un promedio de 1,2. Al comparar entre cepas, la cepa Aan17 proveniente de Nariño, obtuvo el mayor número de generaciones con 1,4.

En cuanto al tiempo de duplicación celular (td), se observaron valores que oscilaron desde 0,7 días para la cepa Aac49 en el medio con extracto de raíz, levadura y glucosa (M1), hasta 2,6 días para la cepa Aan17 en el medio extracto de raíz, levadura y sacarosa (M2), existiendo diferencias altamente significativas entre tratamientos y cepas. En coherencia con $\mu$, el menor td se presentó en el medio de cultivo con extracto de raíz, levadura y glucosa (M1) con un tiempo de duplicación celular de 33,93 h; mientras que el mayor td se manifestó en el medio extracto de raíz, levadura y melaza (M3) con 51,68 h. Entre las cepas, la cepa Aac49 mostró el menor td con $34.9 \mathrm{~h}$.

Existen muy pocos reportes respecto a estos parámetros de crecimiento. Lechevalier \& Lechevalier (1990) y Murry et al. (1984) reportaron un tiempo de duplicación celular entre 2 a 5 días, efecto asociado a su largo periodo de crecimiento, lo cual indica que el uso de medios nutritivos con la inclusión de levadura y de extracto de raíz, sin importar la fuente de carbono utilizada, permite una reducción del td de 179,8\%. De igual manera, dependiendo de la fuente de carbono, se presentaron reducciones de td en $257 \%$, $142,7 \%$ y $138 \%$ en los medios con glucosa (M1), con sacarosa (M2) y con melaza (M3), respectivamente.

El uso de los diferentes medios de cultivo afectó significativamente el tiempo de duración de la fase exponencial de las tres cepas $(\mathrm{P}<.0001)$. En promedio, el uso del medio M3 (con melaza), estuvo asociado con mayor duración de esta fase (51.68 h), mientras que con el medio con glucosa se presentó el menor tiempo de duración de esta fase (33.93 h).

El rendimiento de biomasa estimada por proteína microbial en relación al sustrato $\left(\mathrm{Y}_{\mathrm{x} / \mathrm{s}}\right)$ presentó diferencias altamente significativas entre los tratamientos. El mayor rendimiento entre medios se observó en el medio M1 con melaza con $1.95 \mathrm{~g} /$ proteína/g sustrato y entre cepas en la cepa control Aaclll con $1.95 \mathrm{~g} /$ proteína/g sustrato. El menor rendimiento se presentó en el medio con glucosa (1.59 g/proteína/g sustrato). Este resultado indica que la melaza permite una biosíntesis de proteína, efecto asociado a la diversidad de metabólica que permite que algunas cepas de Frankia puedan utilizar diferentes fuentes de energía presentes en el medio.

La velocidad específica de consumo $\mathrm{q}_{\mathrm{s}}$ presentó diferencias altamente significativas entre los sustratos y entre cepas. La mayor velocidad se presentó en el medio M1 con glucosa como sustrato con un valor de 0.016 , lo cual es un resultado de esperarse, por cuanto es la fuente de carbono más fácil de utilizar para las cepas. En este parámetro, la cepa proveniente de Nariño Aan17 obtuvo el mayor valor, siendo $143 \%$ y $100 \%$ más alto que el de las cepas Aac49 y Aaclll. 


\section{Conclusiones}

El desarrollo de esta investigación permitió establecer que existe un efecto de la composición del medio de cultivo sobre las curvas de crecimiento de Frankia. La mayor biomasa celular fue obtenida en el medio compuesto de extracto de raíz, extracto de levadura y melaza.

Las mejores respuestas en los parámetros cinéticos se presentaron en los medios con glucosa, con mayor velocidad específica de crecimiento y menor tiempo de duplicación celular y en el medio con melaza donde se presentó un mayor tiempo

\section{Literatura citada}

1. Agudelo, C., Ortega, R. \& Hoyos, J. L. (2010). Determinación de parámetros cinéticos de dos inóculos lácticos: Lactobacillus plantarum A6 y bacterias ácido lácticas de yogurt. Facultad de Ciencias Agropecuarias. Vol. 8(2): 8-16.

2. Anderson, M. D. (2011). Sources of variation in the symbiotic association between Alnus and Frankia in interior Alaska. Presented to the Faculty of the University of Alaska Fairbanks in Partial Fulfillment of the Requirements for the Degree of Doctor of Philosophy. Fairbanks, Alaska, 206p.

3. Beauchemin, N. J., Furnholm, T., Lavenus, J., Svistoonoff, S. Doumas, P., Bogusz, D., Laplaze, L. \& Tisaa, L. (2011). Casuarina root exudates alter the physiology, surface properties, and plant infectivity of Frankia sp. strain Ccl3. Applied and Environmental Microbiology 575-580.

4. Benoist, P., Muller, A., Diem, H. G. \& Schwenckel, J. (1992). High-molecular-mass multicatalytic proteinase complexes produced by the nitrogen-fixing Actinomycete Frankia Strain BR. In. Journal of Bacteriology. Vol. 174 (5): 1495-1504.

5. Benson, D. R.; Vanden Heuvel, B. D. \& Potter, D. (2004). Actinorhizal symbioses: Diversity and biogeography. In. Plant microbiology (ed. M. Gillings). BIOS Scientific Publishers Ltd., Oxford.

6. Boraste, A., Vamsi, K. K., Jhadav, A., Khairnar, Y., Gupta, N., Trivedi, S., Patil, P., Gupta G., Gupta, M., Mujapara, A. K. \& Joshi, B. (2009). Biofertilizers: A novel tool for agriculture. International Journal of Microbiology Research (1):2, 23-31.

7. Broom, D. M., Galindo, F. A. \& Murgueitio, E. (2013). Sustainable, efficient livestock production with high biodiversity and good welfare for animals. Proceedings of the Royal Society B: Biological Sciences 280: 20132025 http://dx.doi.org/10.1098/rspb.2013.2025. de duración de la fase exponencial, mayor velocidad específica de consumo de sustrato y mayor rendimiento celular en base sustrato.

\section{Agradecimientos}

La investigación fue posible gracias a la colaboración del grupo de investigación Agroforestería y Biodiversidad Tropical y a la Corporación Colombiana de Investigación Agropecuaria CORPOICA Tibaitatá.
8. Carú, M., Becerra, A., Sepúlveda, D. \& Cabello, A. (2000). Isolation of infective Frankia strains from root nodules of Alnus acuminata (Betulaceae). World Journal of Microbiology \& Biotechnology. Vol. 16:647-651.

9. Chaia, E. E., Wall, L. G. \& Huss-Danell, K. (2011). Life in soil by the actinorhizal root module endophyte Frankia: a review. Symbiosis 51(3). 201-226.

10. Chamorro, D. \& Rey, A. M. (2008). El componente arbóreo como dinamizado del sistema de producción de leche en el trópico alto Colombiano. Experiencias de Corpoica-Tibaitatá. 2008. En. Murgueitio, Cuartas \& Naranjo (Eds). Ganadería del futuro: investigación para el desarrollo. Fundación CIPAV, Cali, Colombia. Pp 349-398.

11. Chamorro, D. R. \& Rey, A. M. (2005). Potencial de micorrizas, rizobios y Frankia en el establecimiento y manejo de sistemas silvopastoriles. En: Resúmenes de la primera reunión de Red de Recursos Forrajeros. CORPOICA. C.I. Tibaitatá. Pg. 35.

12. Dawson, J. O. (1990). Interactions among actinirhyzal and associated plant species. In: Schwintzer, C: R: \& J. D. Tjepkema (eds.). The biology of Frankia and actinorhyzal plants. San Diego, Academic Press. Pp. 299-316.

13. Doran, P. (1998). Principios de ingeniería de los bioprocesos. Editorial Acribia. Zaragoza, España. 289-291p.

14. Duarte, A. (1998). Introducción a la ingeniería bioquímica. Unidad de publicaciones de la Facultad de Ingeniería. Universidad Nacional de Colombia Sede BogotáColombia. 528p.

15. Gyaneshwar, P., Naresh, G.K., Parekh, L.J. \& Poole, P.S. (2002) Role of soil microorganisms in improving $P$ nutrition of plants. Plant and Soil 245: 83-93.

16. Heath, K.D. \& Tiffin, P. (2007). Context dependence in the coevolution of plant and rhizobial mutualists. Proceedings of the Royal Society B. 274:1905-1912. 
17. Huguet, V., Mergeay, M., Cervantes, E. \& Fernandez, M. (2004). Diversity of Frankia strains associated to Myrica gale in Western Europe: impact of host plant (Myrica vs. Alnus) and of edaphic factors. Environmental of Microbiology. Vol. 6(10):1032-1041.

18. Kazuhiko, T. \& Kozo. T. (1995). Factors affecting the etanol productivity of yeast in molasses. Journal of fermentation and Bioingineering. Vol. 79(5):449-452.

19. Lechevalier, M.P. \& Lechevalier, H.A. (1990). Systematics, isolation and culture of Frankia. In. Schwintzer \& Tjepkem. The Biology of Frankia and actinorhizal plants. New Jersey, USA. Pp 35-60.

20. Lowry, O.H.; Rosenbrough, N.J.\& Randall, R.J. (1951). Protein measuremente with the folin phenol reagent. Journal biology chemistry. 265-275.

21. Murry, M.A., Fontaine, M.S. \& Torrey, J.G. (1984). Growth kinetcs and nitrogenase induction in Frankia sp. HFPArl.3 grown in batch culture. Plant and Soil. Vol. 78:61-78.

22. Nahvi, I., Emtiazi, G. \& Alkabi, L. (2002). Isolation of a flocculating Saccharomyces cerevisiae and investigation of its performance in the fermentation of beet molasses to ethanol. Biomass and Bioenergy. 23: 481-486.

23. Nain, L., Rana, A., Joshi, M., Jadhav, D.S., Kumar, D., Shivay, Y.S., Paul, P. \& Prasann, R. (2010). Evaluation of synergistic effects of bacterial and cyanobacterial strains as biofertilizers for wheat. Plant Soil 331:217-230.

24. Pintor, F.H., Garzón, S.A., Chamorro, D. \& Rey, A.M. (2007). Efecto de la doble inoculación de Frankia y micorrizas arbusculares en aliso (Alnus acuminata) H.B.K en fase de vivero. Trabajo de grado para optar el título de Ing. Agrónomo. UDCA- 120p.

25. Quispel, A. \& Tak, T. (1978). Studies on the growth of the endophyte of Alnus glutinosa (L.) Vill. in nutrient solutions. New Phytologist. Vol. 81:587-600.
26. Quispel, A., Burggraaf, A.J., Borsje, H. \& Tak, T. (1983). The role of lipids in the growth of Frankia isolates. Canadian Journal of Botany Vol. 61:2801-2806.

27. Rediet, O., Mansour, S.R. \& Tisa, L.S. (2013). Effect of salt stress on the physiology of Frankia sp strain Ccl6. Journal Biosci. 38(4): 699-702

28. Rey, A. M. (2006). Estudio de la actividad de cepas nativas de Frankia en Alnus acuminata H.B.K. Tesis de Maestría en Microbiología. Universidad Nacional de Colombia, Facultad de Ciencias. IBUN. 126 p.

29. Romero, M. A. (1996). El Aliso. Alnus acuminata. Proyecto desarrollo forestal campesino en los andes de Ecuador (DFC). Ed. Gráficas Iberia. Quito, Ecuador. 166p.

30. Schubert, M., Koteyeva, N.K., Wabnitz, P.W., Santos, P., Büttner, M., Sauer, N., Demchenko, K. \& Pawlowski, K. (2011). Plasmodesmata distribution and sugar partitioning in nitrogen-fixing root nodules of Datisca glomerata. Planta Vol. 233:139-152.

31. Schultz, N. A. \& Benson, D. R. (1989). Developmental potential of Frankia vesicles. Journal of Bacteriology. Vol. 171(12):6872-6877.

32. Steinfeld, H., Gerner, P., Wassenaar, T., Castel, V., Rosales, M. \& Haan, C. (2009). La larga sombra del Ganado. Problemas ambientales y opciones. (Edición en Español) FAO. Capítulo I. Pp. 2-22.

33. Wani, S. P., Rupela, O. P. \& Lee, K. K. (1995). Sustainable agriculture in the semi-arid tropics through biological nitrogen fixation in grain legumes. Plant and Soil 174: 29-49.

34. Wolters, D.J. (1998). Ineffective Frankia in wet alder soils. Doctor en ciencias naturales y matemáticas. Universidad Agrícola de Wageningen (Holanda). Departamento de ciencias Biomoleculares. 153p. 